\title{
Value analysis of CD69 combined with EGR1 in the diagnosis of coronary heart disease
}

\author{
JIANQIAO PENG $^{1}$ and YI XIANG ${ }^{2}$ \\ ${ }^{1}$ Clinical Laboratory and ${ }^{2}$ Department of Geriatrics, \\ People's Hospital of Hunan Province, Changsha, Hunan 410006, P.R. China
}

Received April 30, 2018; Accepted November 21, 2018

DOI: $10.3892 /$ etm.2019.7175

\begin{abstract}
Expression and clinical significance of CD69 and early growth response (EGR1) in plasma of patients with coronary heart disease (CHD) were investigated. A total of 194 patients with CHD and 130 healthy subjects, respectively, were selected as CHD and control group, clinical data were collected and coronary angiography was performed. RT-qPCR was used to detect the expression of EGR1. Flow cytometry was used to detect the expression level of CD69 and the receiver operating characteristic curve was used to analyze the values of relative expression of CD69 and EGR1. The relative expression of CD69 in plasma of patients with CHD was higher than that in control group, while the relative expression of EGR1 was lower than that in control group. The relative expression of EGR1 in plasma of patients with CHD was negatively correlated with lipoprotein a [Lp(a)] and high sensitive C-reactive protein (hs-CRP) ( $\mathrm{r}=-0.394$ and $-0.524, \mathrm{P}<0.05)$, and the relative expression of CD69 in peripheral blood was positively correlated with [Lp(a)] and hs-CRP ( $\mathrm{r}=0.352$ and $0.402, \mathrm{P}<0.05)$. The area under curve (AUC) of the relative expression of CD69 in peripheral blood of patients with CHD in evaluating the course of the disease of patients was $0.889(95 \%$ CI: 0.822-0.958). The AUC of the relative expression of EGR1 in plasma in evaluating the course of the disease of patients was 0.933 (95\% CI: 0.867-0.978). By the combined detection of CD69 and EGR1, it was found that the AUC was $0.954(95 \%$ CI: 0.887-0.982). The expression level of EGR1 in plasma of patients with CHD decreased, while the expression level of CD69 increased, and both of them were related to the severity of the disease of patients, which could be used as an indicator to evaluate the progression of the patients' conditions.
\end{abstract}

\section{Introduction}

Coronary heart disease (CHD), usually refers to the heart disease caused by myocardial ischemia and hypoxia due to

Correspondence to: Dr Yi Xiang, Department of Geriatrics, People's Hospital of Hunan Province, 61 Jiefang West Road, Changsha, Hunan 410006, P.R. China

E-mail: ete25q@163.com

Key words: CD69, EGR1, coronary atherosclerosis, diagnostic value insufficiency of myocardial blood supply in patients, which is caused by stenosis and obstruction of blood vessel lumen as a result of coronary atherosclerosis (AS) $(1,2)$. AS is mainly caused by the participation of lipid accumulation in the wall of coronary artery and inflammation and immune response (3). The data released by the Statistical Commission of the American Heart Association in 2009 showed (4) that cardiovascular disease has become the top ranking disease in the causes of human death in the world. In China, CHD has become one of the two leading causes of death after malignant tumor, for China has gradually entered an aging society, and the number of elderly people has gradually increased (5). Another study finds (6) that the main risk factors for CHD are age, sex, smoking and diabetes mellitus, and other secondary factors are diet, family history, exercise volume and congenital defects.

CD69 is the earliest expressed signal transduction molecule on the surface of immune system activated T lymphocytes (7). There is a study showing (8) that the downregulation of the expression of CD69 can decrease the activation of $\mathrm{CD}^{+}{ }^{+} \mathrm{T}$ cells in I diabetic mouse model, thus regulating the immune response to the disease. Early growth response (EGR) is an early gene and its family includes four members: EGR1, EGR2, EGR3 and EGR4 (9). EGR can be activated by a variety of extracellular signal molecules to cause cascade amplification of cells, and all genes in the family contain a highly conserved structure domain that binds to DNA. This domain consists of three same zinc finger structures $\left(\mathrm{Cys}_{2} \mathrm{His}_{2}\right)$, which can bind specifically to the downstream promoter region and play a regulatory and transcriptional role (10). EGRl gene is more studied in EGR family and many studies have reported that EGR1 is directly or indirectly involved in the proliferation and differentiation of tumor cells (11). However, CD69 and EGR1 are rarely reported in CHD. We found that EGR1 and CD69 were differentially expressed in CHD by screening from ceo database. Therefore, in this study, the clinical effects of EGR1 and CD69 in CHD were investigated through the detection of the expression of EGR1 and CD69 in the blood of patients with CHD.

\section{Patients and methods}

General data of patients. In the present study, 194 patients with CHD, admitted to the Department of Cardiology in People's Hospital of Hunan Province (Changsha, China) 
from June 2015 to September 2016, were selected as experimental group. Another 130 normal volunteers at the same time in the physical examination center were selected as control group. There were 112 males and 82 females in experimental group, aged from 39-78 years, with an average age of $66.5 \pm 11.3$ years. The patients in experimental group were diagnosed by coronary angiography and all the patients in experimental group were patients with CHD after the examination. There were 70 males and 60 females in control group, aged from 42-80 years, with an average age of $64.8 \pm 10.9$ years. Patients in control group were excluded from CHD and other systemic heart diseases. Biochemical indicators of clinical data of patients were collected, including total cholesterol (TC), triacylglycerol (TG), high density lipoprotein (HDL-C), low density lipoprotein (LDL-C), lipoprotein a [Lp(a)], fasting blood glucose (FBG), glycosylated hemoglobin (HbA1c), high sensitive C-reactive protein (hs-CRP) and serum creatinine (Scr), and statistical analysis was performed.

This study was approved by the Medical Ethics Committee of People's Hospital of Hunan Province (Changsha, China) and patients and their family members were informed and signed the informed consent.

Inclusion and exclusion criteria. Inclusion criteria were: There were $\geq 50 \%$ stenosis in one or more main coronary arteries according to coronary angiography, patient was $>18$ years and course of disease was half a year. Patient had no recent drug treatment, no other hereditary disease, no radiotherapy and chemotherapy, no autism, memory impairment and hearing impairment. Patient cooperated perfectly with follow-up and clinical information.

Exclusion criteria were: Malignant tumors, severe dysfunction of important organs, acute myocarditis and pericarditis, congenital heart disease, immune dysfunction, connective tissue disease, chronic infection, pulmonary embolism and cerebrovascular disease.

Main reagents and instruments. CD69 McAb (mouse anti-human-phycoerythrin labeled), CD3 McAb (mouse anti-human-activated protein), CD4 McAb (mouse anti-human-fluorescein isothiocyanate), PE-labeled mouse IgG1 and CD69 isomorphic control were purchased from BD Biosciences (San Jose, CA, USA). Erythrocyte splitting liquor was purchased from Tiangen Biotech Co., Ltd. (Beijing, China). TRIzol was purchased from Invitrogen; Thermo Fisher Scientific, Inc. (Waltham, MA, USA). Reverse transcriptase and reverse transcription kit were purchased from Takara Biotechnology Co., Ltd. (Dalian, China). 2X SYBR-Green qPCR mix and revert aid first strand cDNA synthesis kit were purchased from Invitrogen; Thermo Fisher Scientific Inc. ABI Prism 7900 PCR instrument was purchased from Thermo Fisher Scientific, Inc.

\section{CD69 detection}

Sample collection and processing. Venous whole blood $(5 \mathrm{ml})$ was extracted with vacuum tubes (EDTA-Na $)_{2}$ on an empty stomach and mixed with Hanks solution at 1:1. Then the mixture was superimposed on the liquid level of $8 \mathrm{ml}$ lymphocyte separating and was centrifuged at $543 \mathrm{x} \mathrm{g}$ for $15 \mathrm{~min}$ at $25^{\circ} \mathrm{C}$. After centrifugation, lymphocytes on the second layer (annular milky white) were collected and put into test tube (containing $10 \mathrm{ml}$ of Hanks solution). Then they were mixed and centrifuged at $543 \mathrm{x}$ g again for $15 \mathrm{~min}$ at $25^{\circ} \mathrm{C}$. The supernatant was discarded, the precipitation was left, and cells were resuspended and washed. Finally, cell concentration was adjusted to $1 \times 10^{6} / \mathrm{ml}$ by $10 \%$ RPMI- 1640 culture medium, and resuspended suspension was added to a 24-orifice plate $(1 \mathrm{ml}$ per orifice). A total of $100 \mu \mathrm{l} / \mathrm{ml}$ for penicillin, streptomycin $(100 \mu \mathrm{l} / \mathrm{ml})$ and PHA $(20 \mu \mathrm{g} / \mathrm{ml})$ for stimulator were added and mixed. The culture was performed $\left(20 \mathrm{~h}\right.$ at $37^{\circ} \mathrm{C}$ and $5 \% \mathrm{CO}_{2}$ incubator), the resuspension was performed by PBS, and the cell concentration was adjusted to $1 \times 10^{6} / \mathrm{ml}$.

Sample detection. Two cell suspensions $(100 \mu \mathrm{l})$ were added to the sample tube. One sample was added to each of the $10 \mu \mathrm{l}$ of CD3, CD4 and CD69 McAbs, respectively, and the other one was added to the same type of CD3 McAb, CD4 McAb and CD69 McAb, respectively. Cell suspensions were incubated in greenhouse for $20 \mathrm{~min}$ and treated without light. After dyeing, $2 \mathrm{ml}$ of erythrocyte splitting liquor was added for mixing. The cell suspensions were static for $10 \mathrm{~min}$ and dissociated without light, and the dissociated test tube was centrifuged at $1,006 \mathrm{xg}$ for $5 \mathrm{~min}$ at $25^{\circ} \mathrm{C}$. The supernatant was discarded, and $2 \mathrm{ml}$ of PBS was added to carry on resuspension of the cells. After centrifugation at $543 \mathrm{x}$ g for $15 \mathrm{~min}$ at $25^{\circ} \mathrm{C}$, the untuberculous antibody was removed, the supernatant was discarded, and $0.5 \mathrm{ml}$ of PBS was added for resuspension. Flow cytometry was used to detect the expression level of $\mathrm{CD} 69$ in $\mathrm{CD}^{+} \mathrm{CD}^{+} \mathrm{T}$ cells.

\section{EGR1 detection}

Sample collection and processing. Venous whole blood $(5 \mathrm{ml})$ was extracted with vacuum tubes (EDTA-Na $)_{2}$ on an empty stomach and centrifuged at $1,006 \mathrm{x} \mathrm{g}$ for $5 \mathrm{~min}$ at room temperature within $30 \mathrm{~min}$ after extraction. Supernatant was discarded, and plasma was transferred to EP tube and stored at $-80^{\circ} \mathrm{C}$ in a refrigerator. Total RNA of frozen plasma was extracted with TRIzol reagent, and extraction procedure was carried out in accordance with the manufacturer's protocol. UV spectrophotometer (L6S; INESA Analytical Instrument Co. Ltd., Shanghai, China) was used to detect the concentration and purity of RNA (the A260/A280 of total RNA solution was within the range of 1.8-2.1), and quality of the total RNA was analyzed by $1 \%$ denatured agarose gel electrophoresis. Reverse transcription procedure was carried out strictly according to the instruction of reverse transcription kit.

$R T-q P C R$ detection. Design and synthesis of EGR1 primers were carried out by Shanghai Bioengineering Co., Ltd., (Shanghai, China) (Table I). PCR reaction kit (2X SYBR-Green qPCR Mix) was used for the configuration of reaction system: A total of $0.2 \mu \mathrm{l}$ for up- and downstream primers respectively, $1 \mu \mathrm{l}$ for cDNA, $5 \mu \mathrm{l}$ for SYBR Select Master Mix and double distilled water added to $10 \mu \mathrm{l}$; ABI Prism 7900 PCR instrument was used for amplification. PrimeScript ${ }^{\mathrm{TM}}$ RT Master Mix (Takara Biotechnology Co., Ltd.) to reverse the total RNA collected according to the kit instructions. Reaction conditions: $2 \mathrm{~min}$ after pre-denaturation at $95^{\circ} \mathrm{C}, 15 \mathrm{sec}$ for denaturation at $95^{\circ} \mathrm{C}, 60 \mathrm{sec}$ for annealing at $60^{\circ} \mathrm{C}, 15 \mathrm{sec}$ for extension 
Table I. Primer sequence.

\begin{tabular}{lcc}
\hline Genes & Upstream & Downstream \\
\hline EGR1 & 5'-CCCTTGCTCCCTTCAATGCT-3' & 5'-CGAAATCCATGGCACAGACAC-3' \\
GAPDH & 5'-AGCCACATCGCTCAGACA-3' & 5'-TGGACTCCACGACGTACT-3'
\end{tabular}

EGR1, early growth response.

at $95^{\circ} \mathrm{C}, 40$ cycles. GAPDH was used as internal reference in this experiment, and was conducted three times. Results were analyzed by $2^{-\Delta \mathrm{Cq}}$ method (12).

Statistical analysis. In the present study, SPSS 20.0 software package (IBM Corp., Armonk, NY, USA) was used for statistical analysis of the collected data, and GraphPad Prism 5 software (La Jolla, CA, USA) was used to plot the resulting data graph. In the present study, the measurement data are expressed by mean \pm standard deviation (mean \pm SD) and tested by t-test. The counting data was expressed by rate (\%), and the comparison between groups was tested by $\chi^{2}$. In the present study, Pearson's correlation analysis was used to analyze the relationships among the variables, and ROC curve was used to plot the two indicators and to evaluate the values of the two indicators in the course of the disease of patients with CHD.

\section{Results}

Comparison of clinical treatment in patients. Through the comparison of the collected data of the two groups, it was found that there was no statistically significant difference in clinical data such as sex, age, smoking and alcohol and in biochemical indicators of TC, TG, LDL-C, FBG and HbAlc ( $\mathrm{P}>0.05)$. However, there were statistically significant differences in HDL-C, [Lp(a)], hs-CRP and Scr between patients in experimental and control group $(\mathrm{P}<0.05)$, and expression level of HDL-C of patients in experimental was significantly lower than that in control group, while the expression level of [Lp(a)], hs-CRP and Scr in experimental was significantly higher than that in control group (Table II).

Expression of CD69 and EGRl in both groups of patients. By comparing the expression of CD69 in two groups by flow cytometry, it was found that the expression of CD69 in experimental group was $16.58 \pm 4.39 \%$, which increased significantly, compared with the control group $10.54 \pm 2.63 \%$, and there was a significant difference in the expression of CD69 between the two groups, which was 1.57 times $(\mathrm{P}<0.05)$. Through the RT-qPCR detection of the expression of EGR1 in plasma, it was found that the expression level of EGR1 (1.255 \pm 0.362$)$ in control group was significantly higher than that in experimental group (0.254 \pm 0.094$)$, and there was a significant difference between the two groups $(\mathrm{P}<0.05)$ (Figs. 1 and 2$)$.

Correlation analysis of the expression and clinical indicators of CD69 and EGR1 in patients with CHD. Through Pearson's correlation analysis, it was found that the expression level of CD69 in peripheral blood of patients with CHD was positively
Table II. Clinical information of patients (n).

\begin{tabular}{|c|c|c|c|c|}
\hline \multirow[b]{2}{*}{ Variables } & \multicolumn{2}{|c|}{ Groups } & \multirow[b]{2}{*}{$t / \chi^{2}$} & \multirow[b]{2}{*}{ P-valu } \\
\hline & $\begin{array}{l}\text { Experimental } \\
\quad(n=194)\end{array}$ & $\begin{array}{l}\text { Control } \\
(n=130)\end{array}$ & & \\
\hline Sex & & & 0.477 & 0.49 \\
\hline Male & 112 & 70 & & \\
\hline Female & 82 & 60 & & \\
\hline Age (years) & & & 0.837 & 0.36 \\
\hline$\geq 55$ & 84 & 63 & & \\
\hline$<55$ & 110 & 67 & & \\
\hline Smoking & & & 0.003 & 0.954 \\
\hline Yes & 120 & 80 & & \\
\hline No & 74 & 50 & & \\
\hline Alcohol & & & 0.338 & 0.561 \\
\hline Yes & 51 & 38 & & \\
\hline No & 143 & 92 & & \\
\hline $\mathrm{TC}(\mathrm{mmol} / \mathrm{l})$ & $1.77 \pm 1.05$ & $1.61 \pm 0.72$ & 1.411 & 0.159 \\
\hline TG (mmol/l) & $4.55 \pm 0.82$ & $4.68 \pm 0.94$ & 1.317 & 0.189 \\
\hline HDL-C (mmol/l) & $1.09 \pm 0.22$ & $1.42 \pm 0.28$ & 11.829 & 0.001 \\
\hline LDL-C (mmol/l) & $2.34 \pm 0.64$ & $2.39 \pm 0.71$ & 0.659 & 0.511 \\
\hline$[\mathrm{Lp}(\mathrm{a})](\mathrm{mg} / \mathrm{l})$ & $283.6 \pm 66.5$ & $235.8 \pm 58.3$ & 8.188 & 0.001 \\
\hline $\mathrm{FBG}(\mathrm{mmol} / \mathrm{l})$ & $5.84 \pm 1.15$ & $5.63 \pm 0.89$ & 1.757 & 0.08 \\
\hline HbA1c $(\%)$ & $6.4 \pm 1.0$ & $6.2 \pm 0.9$ & 1.834 & 0.068 \\
\hline hs-CRP (mg/l) & $5.64 \pm 2.67$ & $3.66 \pm 2.31$ & 6.894 & 0.001 \\
\hline $\operatorname{Scr}(\mu \mathrm{mol} / \mathrm{l})$ & $83.5 \pm 26.4$ & $61.3 \pm 17.6$ & 8.409 & 0.001 \\
\hline
\end{tabular}

TC, total cholesterol; TG, triacylglycerol; HDL-C, high density lipoprotein; LDL-C, low density lipoprotein; [Lp(a)], lipoprotein a; FBG, fasting blood glucose; HbA1c, glycosylated hemoglobin; hs-CRP, high sensitive C-reactive protein; Scr, serum creatinine.

correlated with [Lp(a)] and hs-CRP, while the expression level of EGR1 in patients with CHD was negatively correlated with [Lp(a)] and hs-CRP, and the rest of the clinical indicator tables were not included due to no correlation (Table III).

Diagnostic values of the expression level of CD69 and EGRI in patients with CHD. Through plotting the ROC curve of the data of the two groups, it was found that the AUC of the expression level of CD69 in peripheral blood was 0.889 (95\% CI: $0.822-0.958)$, and the sensitivity was $73.4 \%$, and the specificity was $86.5 \%$. The higher the expression level 


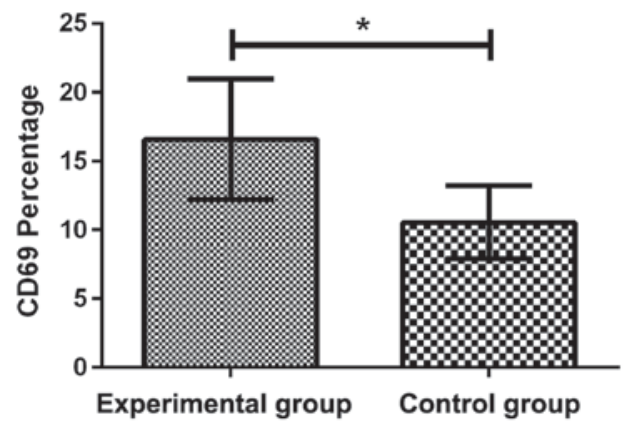

Figure 1. Expression of CD69 in both groups of patients. ${ }^{*} \mathrm{P}<0.05$.

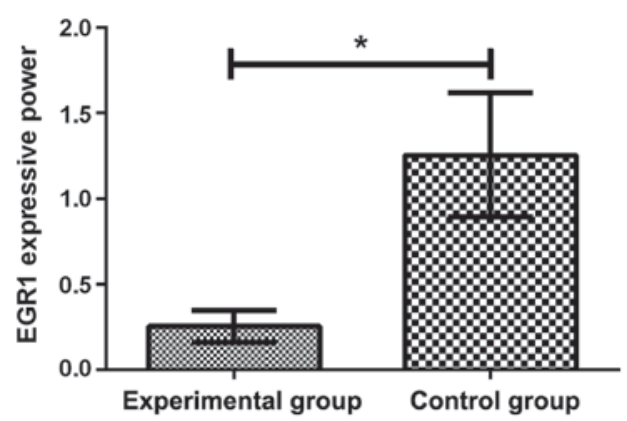

Figure 2. Expression of EGR1 in both groups of patients. EGR1, early growth response. ${ }^{*} \mathrm{P}<0.05$.

Table III. Pearson's correlation analysis of the expression and clinical indicators of CD69 and EGR1.

\begin{tabular}{lcclcc}
\hline CD69 & r value & P-value & EGR1 & r value & P-value \\
\hline Lp(a) & 0.352 & 0.01 & Lp(a) & -0.394 & 0.01 \\
hs-CRP & 0.402 & 0.01 & hs-CRP & -0.524 & 0.01
\end{tabular}

EGR1, early growth response; Lp(a), lipoprotein a; hs-CRP, high sensitive C-reactive protein.

of CD69 was, the higher the sensitivity and specificity were in evaluating the progression of the patient conditions. The AUC of the expression level of EGR1 in plasma was 0.933 (95\% CI: $0.867-0.978$ ), and the sensitivity was $89.7 \%$, and the specificity was $79.2 \%$. The lower the expression level of EGR1 was, the higher the sensitivity and specificity were in evaluating the progression of the condition of the patients. Finally, by the combined detection of CD69 and EGR1, it was found that AUC was 0.954 (95\% CI: 0.887-0.982), and sensitivity was $90.5 \%$, and specificity was $86.3 \%$ (Fig. 3).

\section{Discussion}

As the most common cardiovascular system disease, CHD is mainly caused by stenosis and obstruction of blood vessel lumen as a result of coronary AS. It is one of the major diseases of human health in the world, and statistics show that the incidence of CHD is increasing year by year (13). As the most common clinical cardiovascular disease in the

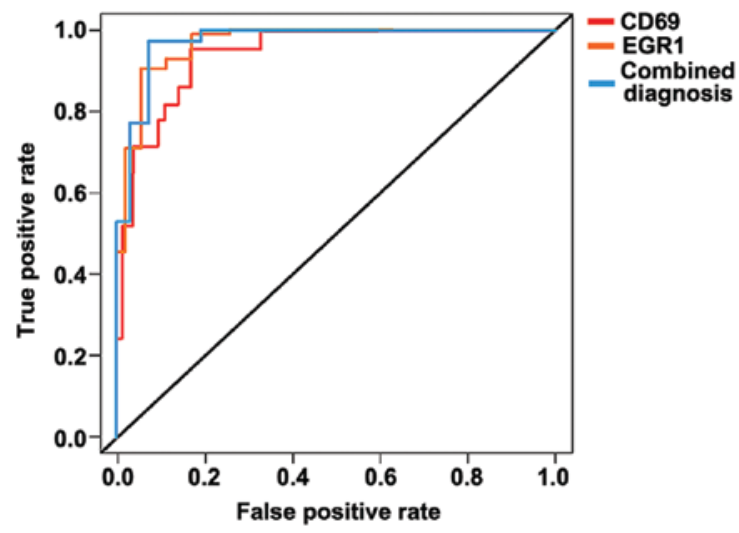

Figure 3. The ROC of CD69 and EGR1. EGR1, early growth response.

elderly, the untimely treatment of CHD will lead to disability and death, and it is also a disease with high mortality in the elderly (14). According to statistics (15), the number of patients suffering from cardiovascular diseases in China is as high as 290 million, accounting for $>30 \%$ of the proportion of death, and the mortality and morbidity rate are increasing year by year. At present, the main diagnostic methods are cardiac stress test and coronary angiography. Cardiac stress test requires higher physical condition of patients, and coronary angiography is one of the most accurate diagnostic methods, but its high price increases the economic burden of many patients (16). Therefore, we need to find new diagnostic indicators to better diagnose the patient's disease and alleviate the patient's suffering by timely treatment to improve the patient's quality of life. In recent years, gene diagnosis of various diseases has become a popular method, and we found that there were differences in the expression level of CD69 and EGR1 in patients with CHD by screening from ceo database.

As a member of type II C plant hemagglutinin-like receptor family, CD69 can be activated by early leukocyte receptor induction, which is less expressed in the resting lymphocytes. After the activation of cells, its expression is induced rapidly, which can be detected on the surface of different activated leukocyte subsets (17). In addition to the immune response, the receptor has many other effects, such as synthesis and differentiation of cells, and regulation of inflammatory response (18). In the present study, through the detection of CD69 in peripheral blood of patients with CHD by flow cytometry, it was found that the expression level of lymphocyte subsets $\mathrm{CD} 69$ in $\mathrm{CD}^{+} \mathrm{CD}^{+} \mathrm{T}$ in peripheral blood of patients with CHD in experimental group was significantly higher than that in control group, which supported the result of gene chip screening. Moreover, in the study of Lei et al (19), it was found that the expression of CD69 could be an independent prognostic factor in patients with type 2 diabetes mellitus and CHD, which also supported the results of this experiment.

EGR1 has been named for existing widely in human cells and its ability to express rapidly. EGR1 belongs to one of the members of the immediate early family, and $>30$ members of the family all have zinc finger structure coding region and have high homology (20). There is a study showing that EGR1 has a complex signal pathway, which plays an important role in cell growth, differentiation, proliferation and inflammatory 
response (21). As a pathological reaction caused by vascular stenosis and hemodynamic obstruction, CHD is composed of many factors such as inflammation, secondary thrombus and plaque rupture (22). In this study, by detecting the expression of EGR1 in plasma of patients, it was found that the relative expression level of EGR1 in plasma of patients in the experimental group decreased significantly, compared with the control group. Besides, in the study of Toutouzas et al (23), it was found that through RT-qPCR detection, the expression of EGR1 of patients with CHD in the blood stasis group and the non-blood stasis group was significantly lower than that in normal group, which also showed that there was a difference in the expression of EGR1 in patients with CHD. We speculated that the decrease of the expression of EGR1 might be due to the change of the patient's condition, which inhibited the binding of zinc finger structure coding region of EGR1 to downstream related genes, leading to the difference of expression of many biochemical factors, so as to result in the occurrence of CHD in patients. As a cholesterol macromolecule lipoprotein, [Lp(a)] plays an important role in the occurrence of CHD and it has been shown to be an independent risk factor for CHD (24). hs-CRP is a new independent prognostic indicator for the diagnosis of CHD in recent years, and its expression level is positively correlated with the pathological changes of CHD (25). In the present study, through Pearson's correlation analysis, it was found that CD69 and EGR1 were correlated with the expression of [Lp(a)] and hs-CRP of patients, which suggested that the expression level of CD69 and EGR1 was related to the severity of patients with $\mathrm{CHD}$.

At the end of the study, the results of ROC curve analysis showed that the AUC of the expression level of CD69 in peripheral blood in the course of the disease of patients was 0.889 (95\% CI: $0.822-0.958$ ), sensitivity was $73.4 \%$, and specificity was $86.5 \%$. AUC of the expression level of EGR1 in plasma in the course of the disease of patients was 0.933 (95\% CI: $0.867-0.978$ ), sensitivity was $89.7 \%$ and the specificity was $79.2 \%$, which also showed that the expression level of the two indicators was expected to be a new indicator for evaluating the progression of the conditions of patients with CHD.

However, the number of patients is small and the patients are all local. Whether there are regional differences in the results needs to be verified by a large number of samples. Therefore, we hope to increase our sample size and regional samples in future studies to prove and perfect the correctness and objectivity of this study.

In conclusion, the expression level of EGR1 in plasma of patients with CHD decreased, while the expression level of CD69 in peripheral blood increased, and both of them were related to the severity of the disease of patients, which could be used as an indicator to evaluate the progression of the condition of the patients.

\section{Acknowledgements}

Not applicable.

\section{Funding}

No funding was received.

\section{Availability of data and materials}

The datasets used and/or analyzed during the present study are available from the corresponding author on reasonable request.

\section{Authors' contributions}

JP designed the study and wrote the manuscript. JP and YX were responsible for sample collection and PCR. Both authors read and approved the final study.

\section{Ethics approval and consent to participate}

The study was approved by the Ethics Committee of People's Hospital of Hunan Province (Changsha, China). Signed informed consents were obtained from the patients or the guardians.

\section{Patient consent for publication}

Not applicable.

\section{Competing interests}

The authors declare that they have no competing interests.

\section{References}

1. García de Tena J: Inflammation, atherosclerosis, and coronary artery disease. N Engl J Med 353: 429-430, author reply 429-430, 2005.

2. Anderson L, Oldridge N, Thompson DR, Zwisler AD, Rees K, Martin N and Taylor RS: Exercise-based cardiac rehabilitation for coronary heart disease: Cochrane systematic review and meta-analysis. J Am Coll Cardiol 67: 1-12, 2016.

3. Catapano AL, Chapman J, Wiklund O and Taskinen MR: The new joint EAS/ESC guidelines for the management of dyslipidaemias. Atherosclerosis 217: 1-44, 2011.

4. Lloyd-Jones D, Adams R, Carnethon M, De Simone G, Ferguson TB, Flegal K, Ford E, Furie K, Go A, Greenlund K, et al; American Heart Association Statistics Committee and Stroke Statistics Subcommittee: Heart disease and stroke statistics 2009 update: A report from the American Heart Association Statistics Committee and Stroke Statistics Subcommittee. Circulation 119: e21-e181, 2009.

5. Chen BJ, Pan ZQ and Su XX: Study on changes of TCM syndrome in patients with coronary heart disease before and after intervention treatment. Zhongguo Zhong Xi Yi Jie He Za Zhi 27: 689-691, 2007 (In Chinese).

6. Pogosova N, Kotseva K, De Bacquer D, von Känel R, De Smedt D, Bruthans J and Dolzhenko M; EUROASPIRE Investigators: Psychosocial risk factors in relation to other cardiovascular risk factors in coronary heart disease: Results from the EUROASPIRE IV survey. A registry from the European Society of Cardiology. Eur J Prev Cardiol 24: 1371-1380, 2017.

7. Mackay LK, Rahimpour A, Ma JZ, Collins N, Stock AT, Hafon ML, Vega-Ramos J, Lauzurica P, Mueller SN, Stefanovic T, et al: The developmental pathway for $\mathrm{CD}_{103}{ }^{+} \mathrm{CD}^{+}$ tissue-resident memory $\mathrm{T}$ cells of skin. Nat Immunol 14: 1294-1301, 2013.

8. Marsolais D, Yagi S and Rosen $\mathrm{H}$ : Activation of sphingosine-1-phosphate receptor 1 inhibits $\mathrm{CD} 4^{+} \mathrm{T}$ cell response in a murine model of allergic airway inflammation. Am J Respir Crit Care Med 179: A4304, 2009.

9. Kumar SS, Tomita Y, Wrin J, Bruhn M, Swalling A, Mohammed M, Price TJ and Hardingham JE: High early growth response 1 (EGR1) expression correlates with resistance to anti-EGFR treatment in vitro and with poorer outcome in metastatic colorectal cancer patients treated with cetuximab. Clin Transl Oncol 19: 718-726, 2017. 
10. Bhattacharyya S, Wu M, Fang F, Tourtellotte W, FeghaliBostwick C and Varga J: Early growth response transcription factors: Key mediators of fibrosis and novel targets for anti-fibrotic therapy. Matrix Biol 30: 235-242, 2011.

11. Sun M, Nie FQ, Zang C, Wang Y, Hou J, Wei C, Li W, He X and Lu KH: The pseudogene DUXAP8 promotes non-small-cell lung cancer cell proliferation and invasion by epigenetically silencing EGR1 and RHOB. Mol Ther 25: 739-751, 2017.

12. Dang Y,Luo D and Rong M: Underexpression of miR-34a in hepatocellular carcinoma and its contribution towards enhancement of proliferating inhibitory effects of agents targeting c-MET. PloS one, 8: e61054, 2013.

13. Satija A, Bhupathiraju SN, Spiegelman D, Chiuve SE, Manson JE, Willett W, Rexrode KM, Rimm EB and Hu FB: Healthful and unhealthful plant-based diets and the risk of coronary heart disease in U.S. adults. J Am Coll Cardiol 70: 411-422, 2017.

14. Blechacz B: Cholangiocarcinoma: Current knowledge and new developments. Gut Liver 11: 13-26, 2017.

15. Zhou M, Wang H, Zhu J, Chen W, Wang L, Liu S, Li Y, Wang L, Liu Y, Yin P, et al: Cause-specific mortality for 240 causes in China during 1990-2013: A systematic subnational analysis for the Global Burden of Disease Study 2013. Lancet 387: 251-272, 2016.

16. Danad I, Szymonifka J, Twisk JWR, Norgaard BL, Zarins CK, Knaapen P and Min JK: Diagnostic performance of cardiac imaging methods to diagnose ischaemia-causing coronary artery disease when directly compared with fractional flow reserve as a reference standard: A meta-analysis. Eur Heart J 38: 991-998, 2017.

17. Kimura MY, Hayashizaki K, Tokoyoda K, Takamura S, Motohashi S and Nakayama T: Crucial role for CD69 in allergic inflammatory responses: CD69-Myl9 system in the pathogenesis of airway inflammation. Immunol Rev 278: 87-100, 2017.

18. Hayashi Y, Okutani M, Ogawa S, Tsukahara T and Inoue R Generation of anti-porcine CD69 monoclonal antibodies and their usefulness to evaluate early activation of cellular immunity by flow cytometric analysis. Anim Sci J 2018. Anim Sci J 89: $825-832,2018$
19. Lei L, Cui L, Mao Y, Zhang X, Jiang Q, Dong S and Wang Y: Augmented CD25 and CD69 expression on circulating CD ${ }^{+}$ $\mathrm{T}$ cells in type 2 diabetes mellitus with albuminuria. Diabetes Metab 43: 382-384, 2017.

20. Ghatak S, Markwald RR, Hascall VC, Dowling W, Lottes RG, Baatz JE, Beeson G, Beeson CC, Perrella MA, Thannickal VJ, et al: Transforming growth factor $\beta 1$ (TGF $\beta 1$ ) regulates CD44V6 expression and activity through extracellular signal-regulated kinase (ERK)-induced EGR1 in pulmonary fibrogenic fibroblasts. J Biol Chem 292: 10465-10489, 2017.

21. Shajahan-Haq AN, Boca SM, Jin L, Bhuvaneshwar K, Gusev Y, Cheema AK, Demas DD, Raghavan KS, Michalek R, Madhavan S, et al: EGR1 regulates cellular metabolism and survival in endocrine resistant breast cancer. Oncotarget 8: 96865-96884, 2017.

22. $\mathrm{Xu} \mathrm{S}$, Bendeck M and Gotlieb AI: Vascular pathobiology: Atherosclerosis and large vessel disease. In: Cardiovascular Pathology. Butany J and Buja M (eds). 4th edition. Academic Press, MA, pp85-124, 2016.

23. Toutouzas K, Colombo A, Stefanadis C: Inflammation and restenosis after percutaneous coronary interventions. Europ Heart J 25: 1679-1687, 2004.

24. Vogt A: Hyperlipoproteinaemia(a) - apheresis and emerging therapies. Clin Res Cardiol Suppl 12 (Suppl 1): 12-17, 2017.

25. Tayefi M, Tajfard M, Saffar S, Hanachi P, Amirabadizadeh AR, Esmaeily H, Taghipour A, Ferns GA, Moohebati M and Ghayour-Mobarhan M: hs-CRP is strongly associated with coronary heart disease (CHD): A data mining approach using decision tree algorithm. Comput Methods Programs Biomed 141: 105-109, 2017.

This work is licensed under a Creative Commons Attribution-NonCommercial-NoDerivatives 4.0 International (CC BY-NC-ND 4.0) License. 